\title{
Spectrum sensing in single channel and multi-channel cognitive radio networks
}

\author{
Amira Osama ${ }^{1}$, Heba A. Tag El-Dien², Ahmad A. Aziz El-Banna ${ }^{3}$, Adly Tag El-Din ${ }^{4}$ \\ ${ }^{1}$ High Institutes for Engineering and Technology Al-obour, Egypt \\ ${ }^{2,3,4}$ Faculty of Engineering at Shoubra, Benha University, Egypt
}

\begin{abstract}
Article Info
ABSTRACT

Article history:

Received Jan 12, 2019

Revised Apr 12, 2019

Accepted May 10, 2019

\section{Keywords:}

Cognitive radio

Spectrum sensing

Throughput channel

Sensing the existence or absence of primary user is the major chore of cognitive radio networks. Nevertheless, Spectrum sensing is the core process of cognitive radio and with target to find idle channels. Various detection techniques exist, however, energy detection is considered as the most used detector because of its lower computational cost. In this paper, we proposed a study of throughput for a cognitive radio system. We had two scenarios, in the first scenario; a study of throughput against probability of false alarm was done; where, only one channel is sensed, to maximize the individual channel throughput. In the second scenario, multi-channel is sensed to maximize the overall system capacity. In addition, different number of channels is considered with different sensing times and at different throughput costs.The performance of the network has been investigated in terms of maximum throughput for optimal number of cognitive radio channels.
\end{abstract}

Copyright () 2019 Institute of Advanced Engineering and Science. All rights reserved.

Corresponding Author:

Amira Osama,

High Institutes for Engineering and Technology Al-obour,

Cairo, Egypt.

Email: amiraosama111@gmail.com

\section{INTRODUCTION}

Cognitive Radio (CR) has been recently proposed as a smart and agile technology which allows illegal users to utilize the licensed bands [1-5]. CR system has two users, Primary User (PU) and Secondary User (SU) [6]. Spectrum management is the main function in CR, it has many process like Spectrum Sensing (SS), spectrum decision and spectrum handoff. SS is one of the major functionality distinguishing CR sensors network from traditional Wireless Sensor Networks (WSNs). Spectrum decision where CR network needs to analyze the sensing data and make decision about the channel and transmission parameters. Spectrum handoff when a primary user arrives again to use a previously available channel, CR node must detect this activity and handoff to another sensed channel within a certain time [7]. To ensure that the operations of the primary users are not affected, it must possess the spectrum-sensing capability.Meanig that, the ability of detecting the presence of primary signals in the bands of interest. Spectrum sensing has many techniques (e.g. Matched filter, energy detection, feature detection, interference temperature) [8]. In this paper, energy detection is used, because it is a common technique for spectrum sensing which is simple and fast, works better in high Signal to Noise Ratio (SNR), but it is not robust at low SNR and cannot differentiate between noise and signal [9].The CR system may be one channel or Multi-channels, each system has advantages and disadvantages, its use depend on what we need. In CR, the secondary users need to speculatively sense the idle channels [10-13]. Once an idle channel is sensed, the secondary system will access this channel. Therefore, spectrum sensing, [14-17] as an essential problem in CR, requests the secondary users to efficiently and effectively detect the presence of the primary signals. There are two measures that related to the sensing performance. First one is the probability of detection, which is the probability of the existence of 
the primary user being detected. Another is the probability of false alarm, which is the probability that the primary user is detected as existence, but actually it is absent [18-25].

Main design principles, potential, advantages, application areas and network architectures of CRSNs are introduced in [1]. In [3] the optimal sensing has been proposed to maximize the channel throughput. The proposed optimal Cooperative Spectrum Sensing (CSS) settings for both the single channel sensing and wide-band sensing are analyzed and calculated in detail by using some simple but reliable methods. In [4] an optimal multiband joint detection method is proposed to increase the performance of secondary user over multiple frequency bands. In [5] the researchers studied the problem of designing the sensing slot duration to maximize the achievable throughput for the secondary users under the constraint that the primary users are sufficiently protected, Using energy detection. In [6] multiband joint detection has proposed for wideband spectrum sensing in CR networks. The basic strategy is to take into account the detection of primary users jointly across a bank of narrowband sub bands rather than considering only one single band at a time. Authors in [8] investigated the performance of spectrum sensing for Wi-Fi network where customer-premises equipment is using CR technology. It is found that the throughput becomes maximum at optimal sensing time. Maximum throughput of the network has been investigated for different number of optimal CR users and maximum throughput decreases as the number of optimal CR users increases. In [13] the change of the average effective capacity has been studied in a single frame with the number of channels under different PU traffic rates. It provides a reference for the establishment of cognitive radio system under the different licensed networks. Authors in [14] presented an optimal multiband sensing-time-adaptive joint detection (MSJD) framework for wideband spectrum sensing. In [20] authors introduced an Orthogonal Frequency Division Multiplexing (OFDM) based cognitive multi relay network is investigated to maximize the transmission rate of the cognitive radio (CR) with enhanced fairness among CR users with interference to the primary users (PUs) being managed below a certain threshold level.

In this paper, we are going to study single and multi-channel throughput, make discussion at both of them and search how to find optimal number of channels in multi-channel system. The rest of this paper is organized as following, Section 2 presents energy detection, single channel and multi-channel, Section 3 discusses simulation results, and finally the paper is concluded in Section 4.

\section{RESEARCH METHOD}

A single and multi-channel are two system models, the first one has single channel with primary users (PUs) and secondary users (SUs), the second one has multi-channels, we study the behave of each system to see how the SU can access the channel without interfering the PU.

The Energy Detection (ED) doesn't need any information about the primary user's signal. It compares the received signal power to the noise power, if the signal power is greater than the pre-assumed noise power level, then the detector decide that the primary signal presence. Without loss of generality, we assume that the noise power is identical at different secondary users. The detection problem can be summarized using two binary hypotheses that can be addressed as $H_{0}$ and $H_{1}$ denote the absence and the presence of the primary user, respectively. Therefore the two hypotheses can be formulated as in [7].

$$
\begin{aligned}
& H_{0}: X(n)=V(n) \\
& H_{1}: X(n)=S(n)+V(n)
\end{aligned}
$$

Where $X(n)$ is the received signal at cognitive radio node, $S(n)$ is the transmitted primary user signal, and $V(n)$ is the noise with variance $\sigma^{2}$.

In single channel, secondary users access to the primary channel only when the channel is sensed to be idle. Let $\delta$ denote the probability that the channel is idle [1]. Therefore, the probability that the channel occupied by only the primary users is $\delta Q_{d}$, the probability that the channel occupied simultaneously by both primary users and secondary users is $\delta\left(1-Q_{d}\right)$ and the probability that the channel is occupied only by the secondary users is $\left(1-Q_{f}\right)(1-\delta)$. Let $T_{p}$ and $T_{p}{ }^{\prime}$ denote the throughput of the primary system without and with the existence of the secondary user, respectively. Let $T_{S}$ and $T_{S}{ }^{\prime}$ denote the throughput of the secondary system without and with the existence of primary user, respectively [1]. Therefore the channel throughput $J$ can be formulated as in [1].

$$
\mathrm{J}=\delta \mathrm{T}_{\mathrm{P}} \mathrm{Q}_{\mathrm{d}}+\delta\left(\mathrm{T}_{\mathrm{p}}{ }^{\prime}+\mathrm{T}_{\mathrm{s}}{ }^{\prime}\right)\left(1-\mathrm{Q}_{\mathrm{d}}\right)+(1-\delta)\left(1-\mathrm{Q}_{\mathrm{f}}\right) \mathrm{T}_{\mathrm{s}}
$$

We can rewrite (3) as 


$$
J=a_{0}+a_{1} Q_{1}-a_{2} Q_{f}
$$

Where the final false-alarm probability $Q_{f}$ and the final detection probability $\left(Q_{d}\right)$. Note that, $a_{0}, a_{1}$ and $a_{2}$ are constants in the optimization problem [1].

In multi-channel, the performance of spectrum sensing has been investigated in terms of optimal number of CR users $\left(n_{\text {opt }}\right)$ in cooperation [8](as every SU represent one channel in our system), throughput $\left(R\left(n_{\text {opt }}, t\right)\right)$ for sensing time and maximum throughput $R_{\text {Max }}\left(n_{\text {opt }}\right)$ for optimal number of CR users. The received signal at each $\mathrm{CR}$ is sampled at sampling frequency fs. Each frame of CR consists of spectrum sensing time $(t)$ and data transmission time $(T-t)$, where $T$ is the total frame time. Let $K$ be the number of samples available during $\tau$. Hence the number of samples, $K=t$.fs $[14,8]$.

$$
\begin{aligned}
& \mathrm{P}_{\mathrm{f}}(\mathrm{t})=\frac{1}{2} \operatorname{erfc}\left(\frac{1}{\sqrt{2}}\left(\frac{\lambda}{\sigma^{2}}-1\right) \sqrt{\mathrm{k}}\right) \\
& \mathrm{P}_{\mathrm{d}}(\mathrm{t})=\frac{1}{2} \operatorname{erfc}\left(\frac{1}{\sqrt{2}}\left(\frac{\lambda}{\sigma^{2}}-\gamma-1\right) \sqrt{\frac{\mathrm{k}}{2 \gamma+1}}\right)
\end{aligned}
$$

Where $\mathrm{V}$ is the SNR at the CR receiver and $\lambda$ is the decision threshold. To ensure reliable sensing performance, detection probability must be high. The decision threshold as in [8],

$$
\lambda=\sigma^{2}\left[\frac{(\sqrt{2}(2 \gamma+1) \operatorname{erfcinv}(2 \mathrm{Pd})+\gamma \sqrt{\mathrm{k}})}{\sqrt{\mathrm{k}}}+1\right]
$$

A CR user can transmit data when the PU is not active. Hence, the CRs can transmit data when the decision goes in favor of false alarm or missed detection. Let us consider nopt that denotes the number of CRs in spectrum sensing. The throughput for the overall false alarm probability is $[8,5]$

$$
R_{0}=\frac{T-\tau}{T} C_{o}\left(1-Q_{\text {fopt }}(t)\right) P\left(H_{o}\right)
$$

Where $C_{0}$ is the throughput in the absence of PU.

The throughput for the overall missed detection probability is

$$
\mathrm{R}_{1}=\frac{\mathrm{T}-\tau}{\mathrm{T}} \mathrm{C}_{1}\left(1-\mathrm{Q}_{\text {dopt }}(\mathrm{t})\right) \mathrm{P}\left(\mathrm{H}_{1}\right)
$$

Where $C_{1}$ is the throughput in the presence of PU.

From (5),(6) we get

$$
\begin{aligned}
& \mathrm{Q}_{\text {fopt }}=1-\left(1-\mathrm{P}_{\mathrm{f}}(\mathrm{t})\right)^{2} \\
& \mathrm{Q}_{\mathrm{dopt}}=1-\left(1-\mathrm{P}_{\mathrm{d}}(\mathrm{t})\right)^{2}
\end{aligned}
$$

From the above discussion we can get total throughput $R\left(n_{o p t}, t\right)$ is a function of $n_{\text {opt }}$ and $t$. Hence $R\left(n_{\text {opt }}, t\right)$ of the CR network can be written as

$$
\mathrm{R}\left(\mathrm{n}_{\mathrm{opt}}, \mathrm{t}\right)=\frac{\mathrm{T}-\mathrm{t}}{\mathrm{T}}\left(\mathrm{C}_{0}\left(1-\mathrm{Q}_{\text {fopt }}(\mathrm{t})\right) \mathrm{P}\left(\mathrm{H}_{0}\right)+\mathrm{C}_{1}\left(1-\mathrm{Q}_{\text {dopt }}(\mathrm{t})\right) \mathrm{P}\left(\mathrm{H}_{1}\right)\right)
$$

For a fixed $n_{\text {opt }}, R\left(n_{\text {opt }}, t\right)$ is concave means there is a maxima of $R\left(n_{\text {opt }}, t\right)$ i.e., $R_{\text {Max }}\left(n_{\text {opt }}\right)$ When $t=t_{\text {opt }}$, the $R\left(n_{\text {opt }}, t\right)$ reaches at $R_{\text {Max }}\left(n_{\text {opt }}\right)$.

We can say that $C$ is indicates the capacity (we can say that, it is cost spend to get high throughput) gained if the primary user in sub-band is absence. Formulated as in [19],

$$
\mathrm{C}=\mathrm{B} \sum_{\mathrm{i}=1}^{\mathrm{n}} \log _{2}\left(1+\frac{\mathrm{c}}{\mathrm{B}} \frac{\mathrm{E}_{\mathrm{b}} \mathrm{h}_{\mathrm{i}}}{\mathrm{N}_{0}}\right)
$$

Where, $E_{b}$ represents the energy of each bit of information, $h_{i}$ represents the fading coefficient of the secondary user, $N_{0}$ represents noise density. 


\section{RESULTS AND ANALYSIS}

In this section, we provide analytical and simulation results to investigate the performance of spectrum sensing with energy detection method in the considered scenarios.

\subsection{Single Channel}

Figure 1 shows result of channel throughput $J$ versus $P_{f}$ at $\lambda=0.2$ in which sensing parameters are given as average $S N R=8 d B$, time bandwidth product $N=10$, number of secondary users $M=4$, and the system parameters $a_{0}=12, a_{1}=7, a_{2}=9$ [3]. For a given $\lambda$, it is clear that when $P_{f}$ reaches 0.2 throughput starts to decrease slowly till $P_{f}$ reaches 0.4 , throughput begins to decrease with higher rate.

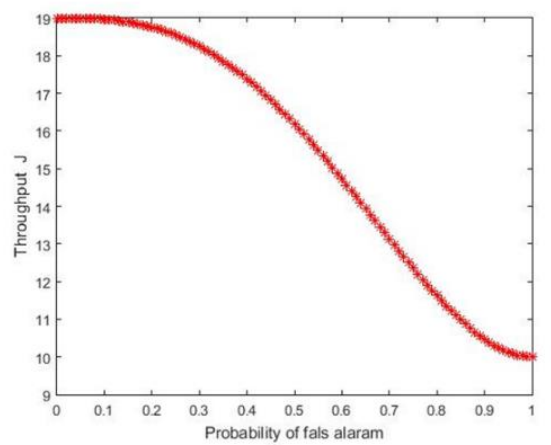

Figure 1. Throughput vs probability of false alarm

\subsection{Multi-Channel}

It is considered that the primary user is using BPSK modulation to transmit its data with $3 \mathrm{MHz}$ bandwidth. The maximum time for which the secondary network unaware of the primary activity is chosen such that $f_{S} \cdot T=3000$ [4]. The frame time of detection cycle is $100 \mathrm{~ms}$ and target detection probability is 0.7 . We choose $P\left(H_{0}\right)=0.8, P\left(H_{1}\right)=0.2, C_{0}=6.6582$ and $C_{1}=6.6137$.

Figure 2 by comparison with [13] we obtained that at $t=3 \mathrm{~ms}, P_{f}$ in reference system reaches to 0.5 but in our proposed system that we improved, $P_{f}$ reduces to 0.15 . This means that improvement of the system is by $30 \%$.

Figure 3 shows the throughput as a function of sensing time for $\mathrm{SNR}=10 \mathrm{~dB}, P_{d}=0.7$. In this case at $\mathrm{n}=15$, It is found that as the sensing time increased, the throughput increased until $t=2 \mathrm{~ms}$, after that throughput is stopped increasing with increasing time.

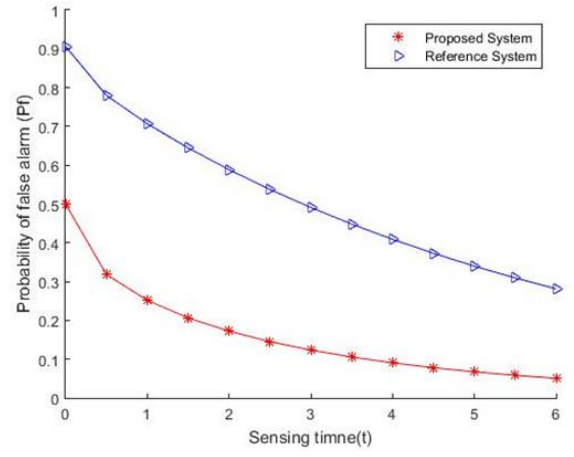

Figure 2. Sensing time vs probability of false alarm

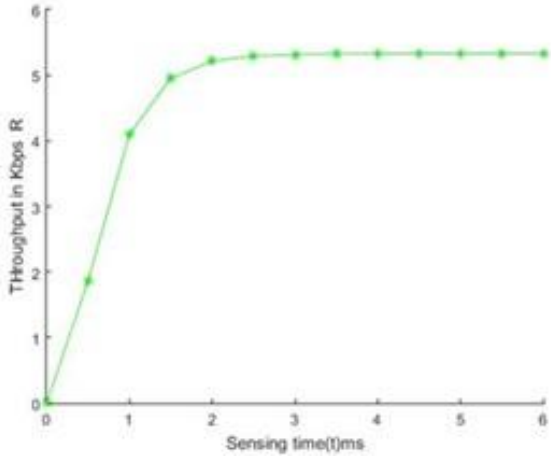

Figure 3. Throughput vs sensing time

Figure 4 shows throughput for network at optimal number of CRs has been compared when $\mathrm{n}=[1,5,10,15]$. We obtained that as the probability of false alarm increases, the throughput of CR network decreases. Hence there is an optimal sensing time in each case for which the throughput is maximum. When 
$P_{f}=0.15$ and $\mathrm{n}=5$, the throughput $\mathrm{R}=2.3$. At $\mathrm{n}=10$ and the throughput $\mathrm{R}=1$, which means that when $\mathrm{n}$ is doubled, the throughput decreased by $43 \%$.By comparing throughput at $P_{f}=0.2$ when $\mathrm{n}=1$, we obtain that throughput is higher in single channel than in multi-channel, it is a logical case.

Figure 5 shows the throughput as a function of time with different costs, working at $n=15$. We obtain that when we increase the cost the peak value of throughput is increased. When $\mathrm{t}=2 \mathrm{~ms}$, at $\mathrm{C}=9.9672$ and the throughput $\mathrm{R}=7.9$, at $\mathrm{C}=6.6582$ and the throughput $\mathrm{R}=5$. This means improving throughput by $63 \%$ with spending added cost $66 \%$.

Figure 6 shows the effect of varying cost at different number of CRs, working at $t=0.2 \mathrm{~ms}$. We obtain varying throughput as $\mathrm{n}$ (number of CRs) increases throughput decreases. When $\mathrm{n}=5$, at $\mathrm{C}=9.9672$ and the throughput $\mathrm{R}=3.1$, at $\mathrm{C}=6.6582$ and the throughput $\mathrm{R}=2.2$. This means improving throughput by $71 \%$ with spending added cost by $65 \%$.

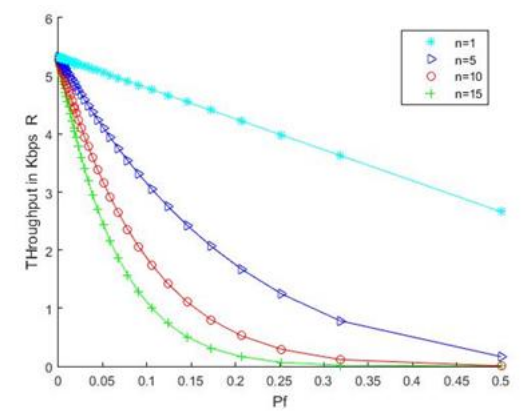

Figure 4. Throughput vs probability of false alarm

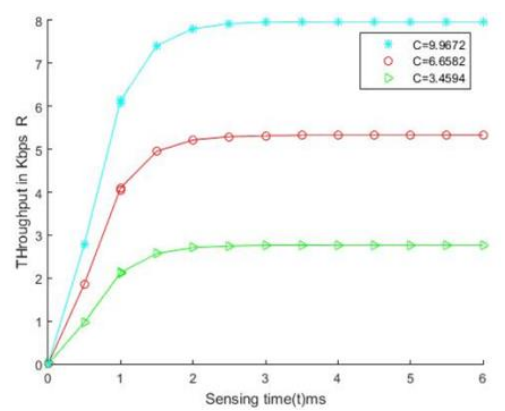

Figure 5. Throughput vs sensing time at different $\mathrm{C}$

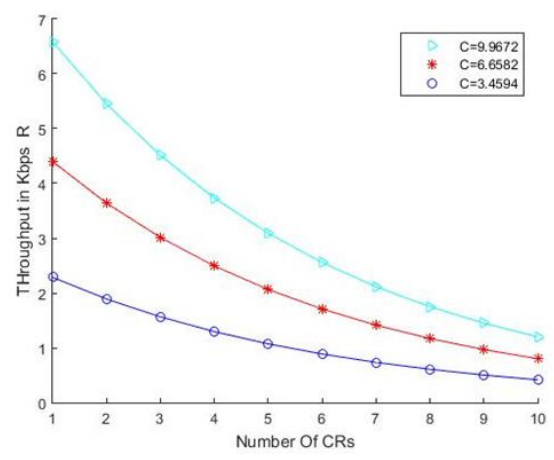

Figure 6. Throughput vs number CRs

\section{CONCLUSION}

The optimal sensing settings to maximize the channel throughput, which is one of great practical interest than other sensing objectives, have been proposed in our work. The proposed optimal settings for both the single channel sensing and wide-band sensing is analyzed and calculated in detail by using some simple but reliable methods. We compared between single channel and multi-channel in throughput to get maximum throughput in both systems. It is an improved system because our target in cognitive radio to have minimum probability of false alarm, our proposed system decreased the probability of false alarm by $30 \%$. From result, when we increase the cost we increase throughput by $63 \%$ to $71 \%$ depending on number of CRs. Theoretical analysis and simulation results have been shown that the proposed optimal settings can substantially improve the channel throughput in cognitive radio networks.

\section{REFERENCES}

[1] Ozgur B. Akan, Osman B. Karli, and Ozgur Ergul, "Cognitive Radio Sensor Networks," IEEE Networ, 09(25): 34-40, 2009.

[2] Dian-Wu Yue, Francis C. M. Lau, and Qian Wang, "Log-Average-SNR Ratio and Cooperative Spectrum Sensing," IEEE Journal of Communications And Networks 18(3):311-319, 2016. 
[3] Juynang Shen, Tao Jiang, Siyang Liu, and Zhongshan Zhang, "Maximum Channel Throughput via Cooperative Spectrum Sensing in Cognitive Radio Networks," IEEE Transactions on Wireless Communications, 8(10): 5166-5175, 2009.

[4] E. Saraniya, B. LakshmiPriya, "Performance Optimization of Cognitive Radio with Wideband Spectrum Sensing," IEEE ICICES, Tamil Nadu, 1-5, 2014.

[5] Ying-Chang Liang, Yonghong Zeng, Edward C.Y. Peh, Anh Tuan Hoang, "Sensing-Throughput Tradeoff for Cognitive Radio Networks," IEEE Transactions on Wireless Communications, 07(04):1326-1337, 2008.

[6] Zhi Quan, Shuguang Cui, Ali H. Sayed, H. Vincent Poor, "Optimal Multiband Joint Detection for Spectrum Sensing in Cognitive Radio Networks," IEEE Transactions on Signal Processing, 57(3):1128-1140, 2009.

[7] Heba A.Tag El-Dien, Rokaia M. Zaki, Mohsen M. Tantawy, Hala M. Abdel-Kader, "Noise Uncertainty Effect on a Modified Two-Stage Spectrum Sensing Technique," TELKOMNIKA Indonesian Journal of Electrical Engineering, 1(2):341-348, 2014

[8] Abhijit Bhowmick, Mrinal K. Das, Joydeep Biswas, Sanjay Dhar Roy and Sumit Kundu, "Throughput Optimization with Cooperative Spectrum Sensing in Cognitive Radio Network," IEEE International Advance Computing Conference (IACC), Gurgaon, 329-332, 2014.

[9] Yujun Chu, Shouyin Liu, "Hard Decision Fusion Based Cooperative Spectrum Sensing over Nakagami-m Fading Channels," IEEE, 12(31):1-4, 2012.

[10] Hongjuan Li, Xiaoshuang Xing, Junda Zhu, Xiuzhen Cheng, Keqiu Li, Rongfang Bie, and Tao Jing, "Utility-Based Cooperative Spectrum Sensing Scheduling in Cognitive Radio Networks,” IEEE, 1-12, 2015.

[11] Sayantan Chowdhury, Puspal Chatterjee, Amitava Mukherjee, Mrinal K. Naskar, "A Throughput-efficient Cooperative Sensing and Allocation Model for Cognitive Radio Networks," IEEE ANTS, 1-3, 2015.

[12] Abhijit Bhowmick, S.D. Roy and Sumit Kundu, "A Hybrid Cooperative Spectrum Sensing for Cognitive Radio Networks in Presence of Fading," NCC IEEE, Mumbai, 1-5, 2015.

[13] Yulong Gao, Ning li, Jiayan Zhang, Kang Xu, "Effective Capacity of Cognitive Radio Systems," ICSP IEEE, Weihai, 1757-1761, 2016.

[14] Pedram Paysarvi-Hoseini, Norman C. Beaulieu, "Optimal Wideband Spectrum Sensing Framework for Cognitive Radio Systems," IEEE Transactions on Signal Processing, 57(3):1128-1140, 2011.

[15] Shaojie Zhang, Abdelhakim Senhaji Hafid, Haitao Zhao, "Cross-layer Rethink on Sensing-throughput Tradeoff for Multi-channel Cognitive Radio Networks," IEEE Transactions on Wireless Communications. 2016, DOI 10.1109/TWC.2016.2592959:1-15.

[16] Mahdi H. Al-Badrawi, Nicholas J. Kirsch, Bessam Z. Al-Jewad, "An Intrinsic Mode Function Based Energy Detector for Spectrum Sensing in Cognitive Radio. International Conference on Computing," Networking and Communications (ICNC) IEEE, USA, 1-6, 2017.

[17] Roshdy Abdelrassoul, Eman Fathy, Mohamed Saad Zghloul, "Comparative Study of Spectrum Sensing for Cognitive Radio System Using Energy Detection over Different Channels," World Symposium on Computer Applications and Research, IEEE. Cairo. 2016:DOI 10.1109/WSCAR.2016.13:32-35.

[18] Ping-Rong Lin, Yin-Zong Chen, Po-Hao Chang, Shiann-Shiun Jeng. Cooperative Spectrum Sensing and Optimization on Multi-Antenna Energy Detection in Rayleigh Fading Channel. the 27th Wireless and Optical Communications Conference IEEE. Hualien.2018: 978-1-5386-4959-6/18:1-5.

[19] Zhang Naiqian, Li Xinyan,Jin Libiao, "Approximation Shannon Limit Spectrum Aggregation Algorithm in Cognitive Radio Networks,” IEEE.2017; 978-1—5386-0497-7:377-380.

[20] Pradip Varade, Akanksha Wabale, Ravinder Yerram, Rupesh Jaiswal, "Throughput Maximization of Cognitive Radio Multi Relay Network with Interference Management," IJECE International Journal of Electrical and Computer Engineering, 8. (2088-8708):2230-2238, 2018.

[21] Z. Quan, S. Cui, A. Sayed, and H. V. Poor, "Optimal multiband joint detection for spectrum sensing in dynamic spectrum access networks,” IEEE Trans, Signal Processing, 57.1128-1140, 2009.

[22] Lin J, Shen L, Bao N, et al., "Channel Characteristic Aware Spectrum Aggregation algorithm in Cognitive Radio networks," Local Computer Networks IEEE, 9. (2008-8778):2222-2230, 2011.

[23] Huang F, Wang W, Luo H, et al., "Prediction-Based Spectrum Aggregation with Hardware Limitation in Cognitive Radio Networks,” IEEE Vehicular Technology Conference, Vtc Spring 2010; 7. (3008-9768):3222-3232.

[24] Q. Zou, S. Zheng, and A. H. Sayed, "Cooperative sensing via sequential detection," IEEE Transactions on Signal Processing, 58. 6266-6283, 2010.

[25] Mansi Subhedar1 and Gajanan Birajdar, "Spectrum sensing Techniques in Cognitive Radio Networks. A survey," $(I J N G N), 3.5277-5288,2011$ 